ALEA, Lat. Am. J. Probab. Math. Stat. 18, 1517-1528 (2021)

DOI: $10.30757 /$ ALEA.v18-55

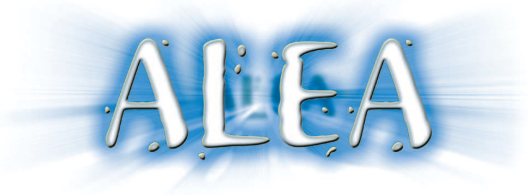

\title{
The giant component of the directed configuration model revisited
}

\author{
Xing Shi Cai and Guillem Perarnau \\ Duke Kunshan University, Kunshan, China \\ E-mail address: xingshi.cai@dukekunshan.edu.cn \\ URL: https://newptcai.gitlab.io/ \\ IMTech, Universitat Politècnica de Catalunya, and Centre de Recerca Matemàtica, Spain \\ E-mail address: guillem.perarnau@upc.edu \\ URL: https://web.mat.upc.edu/guillem.perarnau/
}

\begin{abstract}
We prove a law of large numbers for the order and size of the largest strongly connected component in the directed configuration model. Our result extends previous work by Cooper and Frieze (2004).
\end{abstract}

\section{Introduction and notations}

An SCC (strongly connected component) in a digraph (directed graph) is a maximal sub-digraph in which there exists a directed path from every node to every other node. In this short note, we analyse the size of the giant component, i.e., the largest SCC, in the directed configuration model. This is a continuation of our previous work (Cai and Perarnau, 2020), which studied the diameter of the model.

We introduce the model and our assumptions. For further discussions and references, see Cai and Perarnau (2020). Let $[n]:=\{1, \ldots, n\}$ be a set of $n$ nodes. Let $\overrightarrow{\mathbf{d}}_{n}=\left(\left(d_{1}^{-}, d_{1}^{+}\right), \ldots,\left(d_{n}^{-}, d_{n}^{+}\right)\right)$ be a bi-degree sequence with $m_{n}:=\sum_{i \in[n]} d_{i}^{+}=\sum_{i \in[n]} d_{i}^{-}$. The directed configuration model, $\overrightarrow{\mathbb{G}}_{n}$, is the random directed multigraph on $[n]$ generated by giving $d_{i}^{-}$in half-edges (heads) and $d_{i}^{+}$out half-edges (tails) to node $i$, and then pairing the heads and tails uniformly at random.

Let $D_{n}=\left(D_{n}^{-}, D_{n}^{+}\right)$be the degrees (number of tails and heads) of a uniform random node. Let $n_{k, \ell}$ be the number of $(k, \ell)$ in $\overrightarrow{\mathbf{d}}_{n}$. Let $\Delta_{n}=\max _{i \in[n]} \max \left\{d_{i}^{-}, d_{i}^{+}\right\}$. Consider a sequence of bi-degree sequences $\left(\overrightarrow{\mathbf{d}}_{n}\right)_{n \geq 1}$. Throughout the paper, we will assume the following condition is satisfied,

Condition 1.1. There exists a discrete probability distribution $D=\left(D^{-}, D^{+}\right)$on $\mathbb{Z}_{\geq 0}^{2}$ with $\lambda_{k, \ell}:=$ $\mathbb{P}(D=(k, \ell))$ such that

Received by the editors April 13th, 2020; accepted April 22th, 2021.

2010 Mathematics Subject Classification. 60C05.

Key words and phrases. Random graphs, random directed graphs, configuration model, strongly connected component.

The second author is supported by the Spanish Ministerio de Economía y Competitividad project MTM201782166-P and the MSCA-RISE-2020-101007705 - 'RandNet'. 
(i) $D_{n}$ converges to $D$ in distribution: $\lim _{n \rightarrow \infty} \frac{n_{k, \ell}}{n}=\lambda_{k, \ell}$ for every $k, \ell \in \mathbb{Z}_{\geq 0}$;

(ii) the expectation of $D_{n}$ converges to $D$ the expectation of each marginal of $D$ is finite:

$$
\lim _{n \rightarrow \infty} \mathbb{E}\left[D_{n}^{-}\right]=\lim _{n \rightarrow \infty} \mathbb{E}\left[D_{n}^{+}\right]=\mathbb{E}\left[D^{-}\right]=\mathbb{E}\left[D^{+}\right]=: \lambda \in(0, \infty) ;
$$

(iii) the second moment of $D_{n}$ converges to $D$ and all the second moments are finite: for $i, j \in \mathbb{Z}_{\geq 0}$, $i+j=2$,

$$
\lim _{n \rightarrow \infty} \mathbb{E}\left[\left(D_{n}^{-}\right)^{i}\left(D_{n}^{+}\right)^{j}\right]=\mathbb{E}\left[\left(D^{-}\right)^{i}\left(D^{+}\right)^{j}\right]<\infty
$$

To state the main result, some parameters of $D$ are needed. Let

$$
\nu:=\frac{\mathbb{E}\left[D^{-} D^{+}\right]}{\lambda}<\infty
$$

where the inequality follows from conditions (ii) and (iii). Let $f(z, w):=\sum_{i, j \geq 0} \lambda_{i, j} z^{i} w^{j}$ be the bivariate generating function of $D$. Let $s_{-}$and $s_{+}$be the survival probabilities of the branching processes with offspring distributions which have generating functions $\frac{1}{\lambda} \frac{\partial f}{\partial w}(z, 1)$ and $\frac{1}{\lambda} \frac{\partial f}{\partial z}(1, w)$ respectively. In other words, $\rho_{-}:=1-s_{-}$and $\rho_{+}:=1-s_{+}$are, respectively, the smallest positive solutions to the equations

$$
z=\frac{1}{\lambda} \frac{\partial f}{\partial w}(z, 1), \quad w=\frac{1}{\lambda} \frac{\partial f}{\partial z}(1, w) .
$$

Let $\mathcal{G}_{n}$ be the largest SCC in $\overrightarrow{\mathbb{G}}_{n}$. (If there is more than one such SCC, we choose an arbitrary one among them as $\mathcal{G}_{n}$.) Let $v\left(\mathcal{G}_{n}\right)$ be the number of nodes in $\mathcal{G}_{n}$. Let $e\left(\mathcal{G}_{n}\right)$ be the number of edges in $\mathcal{G}_{n}$. Our main result is the following theorem on $\mathcal{G}_{n}$ :

Theorem 1.2. Suppose that $\left(\overrightarrow{\mathbf{d}}_{n}\right)_{n \geq 1}$ satisfies Condition 1.1. If $\nu>1$, then

$$
\begin{gathered}
\frac{v\left(\mathcal{G}_{n}\right)}{n} \rightarrow \eta<\infty, \\
\frac{e\left(\mathcal{G}_{n}\right)}{n} \rightarrow \lambda s_{-} s_{+}<\infty,
\end{gathered}
$$

in probability, where

$$
\eta:=\sum_{i, j \geq 0} \lambda_{i, j}\left(1-\rho_{-}^{i}\right)\left(1-\rho_{+}^{j}\right)=1+f\left(\rho_{-}, \rho_{+}\right)-f\left(\rho_{-}, 1\right)-f\left(1, \rho_{+}\right) .
$$

If $\nu<1$, then for all $a_{n}$ with $a_{n} \rightarrow \infty$

$$
\frac{v\left(\mathcal{G}_{n}\right)}{a_{n}} \rightarrow 0
$$

in probability.

Remark 1.3. Under Condition 1.1, the probability that $\overrightarrow{\mathbb{G}}_{n}$ is simple is bounded away from 0 , see Blanchet and Stauffer (2013); Janson (2009). Thus Theorem 1.2 holds for a uniform random simple digraph with degree sequence $\overrightarrow{\mathbf{d}}_{n}$.

The two cases $\nu<1$ and $\nu>1$ are often referred to as subcritical and supercritical regimes. As shown in Cai and Perarnau (2020), in the supercritical case, $s_{ \pm}>0$ and $\eta>0$. In other words, whp (with high probability), the size of the largest SCC is less than $\varepsilon n$ for all $\varepsilon>0$ in the first case and linear in $n$ in the second case.

Equation (1.5) in Theorem 1.2 was first proved by Cooper and Frieze (2004) under stronger conditions including $\mathbb{E}\left[\left(D_{n}^{+}\right)^{2} D_{n}^{-}\right]=o\left(\Delta_{n}\right), \mathbb{E}\left[\left(D_{n}^{-}\right)^{2} D_{n}^{+}\right]=o\left(\Delta_{n}\right)$ and $\Delta_{n}=o\left(n^{1 / 12}\right)$. Graf (2016, Theorem 4.1) extended the existence of a linear order SCC provided that $\mathbb{E}\left[D_{n}^{+} D_{n}^{-}\right]$converges uniformly and $\Delta_{n}=o\left(n^{1 / 4}\right)$. Condition 1.1 only implies that $\Delta_{n}=o(\sqrt{n})$, see Cai and Perarnau (2020, Corollary 2.4). In the subcritical case, the results in Cooper and Frieze (2004); Graf (2016) 
only show that whp the largest SCC has order $O\left(\Delta_{n}^{2} \log n\right)$ instead of $O(1)$. A recent paper by Donderwinkel and Xie (2021) considers the critical case $\nu=1$ and shows that the largest SCC rescaled by $n^{1 / 3}$ converges in distribution to a finite graph.

The paper is organized as follows: In Section 2, we study the probability of certain events for branching processes. In Section 3, we recall a graph exploration process defined in Cai and Perarnau (2020) and extend it. Section 4 studies the probability that a set of half-edges reaches a large number of other half-edges. Section 5 shows that the number of nodes which can reach and can be reached from many nodes is concentrated around its mean. Then in Section 6 we show that these nodes form the giant. Finally in Section 7 we give an application of Theorem 1.2 to binomial random digraphs.

\section{Branching processes}

Let $\xi$ be a random variable on $\mathbb{Z}_{\geq 0}$. Let $h_{\xi}$ be the generating function of $\xi$ and $\nu_{\xi}:=h_{\xi}^{\prime}(1)=\mathbb{E}[\xi]$. Let $\left(X_{t}\right)_{t \geq 0}$ be a branching process with offspring distribution $\xi$. If $X_{t}>0$ for all $t \in \mathbb{N}$, then the branching process is said to survive; otherwise, it is said to become extinct. The following are wellknown in branching process theory (see, e.g., van der Hofstad, 2017, Theorem 3.1 and Athreya and Ney, 1972, Theorem I.10.3, respectively):

Lemma 2.1. Let $\rho_{\xi}$ be the smallest nonnegative solution of $z=h_{\xi}(z)$. The survival probability is

$$
s_{\xi}:=\mathbb{P}\left(\cap_{t \geq 1} X_{t}>0\right\}=1-\rho_{\xi} .
$$

Moreover, $s_{\xi}>0$ if and only if $\nu_{\xi}>1$.

Lemma 2.2. Assume that $\nu_{\xi} \in(1, \infty)$. Then there exists a sequence $\left(m_{\xi, t}\right)_{t \geq 0}$ for which $m_{\xi, t}^{1 / t} \rightarrow \nu$, such that $X_{t} / m_{\xi, t} \rightarrow W_{\xi}$, where $W_{\xi}$ is a non-negative random variable for which $\mathbb{P}\left(W_{\xi}=0\right)=1-s_{\xi}$ and which is continuously distributed on $(0, \infty)$.

The main result of this section is the following:

Lemma 2.3. Let $\left(X_{t}\right)_{t \geq 0}$ be a branching process with offspring distribution $\xi$ with $\nu_{\xi} \in(1, \infty)$. Let

$$
T_{\omega}:=\inf \left\{t: X_{t} \geq \omega\right\} .
$$

Then for all $\varepsilon>0$ and as $\omega \rightarrow \infty$,

$$
\mathbb{P}\left(T_{\omega} \leq(1+\varepsilon) \log _{\nu_{\xi}} \omega\right) \rightarrow s_{\xi}
$$

Proof: Let $t_{1}=\left\lfloor(1+\varepsilon) \log _{\nu_{\xi}} \omega\right\rfloor+1$. It suffices to show that $\mathbb{P}\left(T_{\omega}>t_{1}\right) \rightarrow \rho_{\xi}:=1-s_{\xi}$. We split this probability into

$$
\mathbb{P}\left(T_{\omega}>t_{1}\right)=\mathbb{P}\left(\left\{T_{\omega}>t_{1}\right) \cap\left\{X_{t_{1}}=0\right\}\right)+\mathbb{P}\left(\left\{T_{\omega}>t_{1}\right\} \cap\left\{X_{t_{1}} \in(0, \omega)\right\}\right)=: I_{1}+I_{2} .
$$

By Theorem 3.4 of Cai and Perarnau (2020), there exist constants $C>0$ and $\hat{\nu} \in(0,1)$ (both depending only on $\xi$ ) such that for all $\varepsilon>0$,

$$
I_{2}=\mathbb{P}\left(\cap_{i=0}^{t_{1}} X_{i} \in(0, \omega)\right) \leq C \hat{\nu}^{(1+\varepsilon) \log _{\nu_{\xi}} \omega-(1+o(1)) \log _{\nu_{\xi}} \omega-1} \leq C \hat{\nu}^{(\varepsilon / 2) \log _{\nu_{\xi}} \omega}=o(1) .
$$

Let $Y_{t}=\sum_{i=0}^{t} X_{i}$. Let $E$ denote the event that $\left(X_{t}\right)_{t \geq 0}$ becomes extinct, i.e., $X_{t}=0$ for some $t \in \mathbb{N}$. If $\rho_{\xi}=\mathbb{P}(E)=0$, then $I_{1}=0$ and we are done. Thus we can assume that $\rho_{\xi}>0$. Then

$$
I_{1} \leq \mathbb{P}\left(\left\{Y_{t_{1}} \leq\left(1+t_{1}\right) \omega\right\} \cap\left\{X_{t_{1}}=0\right\}\right) \leq \mathbb{P}\left(Y_{t_{1}} \leq\left(1+t_{1}\right) \omega \mid E\right) \mathbb{P}(E) \rightarrow \mathbb{P}(E)=\rho_{\xi},
$$

since a branching process conditioned on becoming extinct has a finite total progeny.

For a lower bound of $I_{1}$, note that $Y_{t}<\omega$ implies $T_{\omega}>t$. Thus,

$$
I_{1} \geq \mathbb{P}\left(\left\{Y_{t_{1}}<\omega\right\} \cap\left\{X_{t_{1}}=0\right\}\right)=\mathbb{P}\left(Y_{t_{1}}<\omega\right)-\mathbb{P}\left(\left\{Y_{t_{1}}<\omega\right\} \cap\left\{X_{t_{1}}>0\right\}\right) .
$$


Note that

$$
\mathbb{P}\left(Y_{t_{1}}<\omega\right) \geq \mathbb{P}\left(Y_{t_{1}}<\omega \mid E\right) \mathbb{P}(E) \rightarrow \mathbb{P}(E)=\rho_{\xi}
$$

By Theorem 6 of Pakes (1971), there exists a sequence $\left(r_{t}\right)_{t \geq 0}$ with $r_{t}^{1 / t} \rightarrow \nu_{\xi}$ such that for all $x>0$,

$$
\mathbb{P}\left(\frac{Y_{t_{1}}}{r_{t_{1}}}<x \mid X_{t_{1}}>0\right) \rightarrow \mathbb{P}\left(Z_{\xi}<x \mid Z_{\xi}>0\right),
$$

where $Z_{\xi}$ is a non-negative random variable for which $\mathbb{P}\left(Z_{\xi}=0\right)=\rho_{\xi}$ and which has continuous distribution on $(0, \infty)$. Therefore, for all $\delta>0$,

$$
\mathbb{P}\left(Y_{t_{1}}<\omega \mid X_{t_{1}}>0\right) \leq \mathbb{P}\left(\frac{Y_{t_{1}}}{r_{t_{1}}}<\delta \mid X_{t_{1}}>0\right) \rightarrow \mathbb{P}\left(Z_{\xi}<\delta \mid Z_{\xi}>0\right)
$$

as $\omega \rightarrow \infty$. Since $\delta$ is arbitrary, we have

$$
\mathbb{P}\left(Y_{t_{1}}<\omega \mid X_{t_{1}}>0\right) \rightarrow 0 .
$$

Putting (2.11) and (2.8) into (2.7) gives the desired lower bound.

Lemma 2.3 can be generalized to multiple iid branching processes as follows:

Corollary 2.4. Let $\left(X_{1, t}\right)_{t \geq 0}, \ldots,\left(X_{x, t}\right)_{t \geq 0}$ be $x \in \mathbb{N}$ independent branching processes with offspring distribution $\xi$. Assume that $\nu_{\xi} \in(1, \infty)$. Let

$$
T_{\omega}^{(x)}:=\inf \left\{t: \sum_{i=1}^{x} X_{i, t} \geq \omega\right\}
$$

Then for all $\varepsilon>0$ and as $\omega \rightarrow \infty$,

$$
\mathbb{P}\left(T_{\omega}^{(x)} \leq(1+\varepsilon) \log _{\nu_{\xi}} \omega\right) \rightarrow 1-\left(1-s_{\xi}\right)^{x} .
$$

Proof: Let $t_{1}=\left\lfloor(1+\varepsilon) \log _{\nu_{\xi}} \omega\right\rfloor+1$. Let $T_{i, \omega}=\inf \left\{t \geq 1: X_{i, t} \geq \omega\right\}$. By Lemma 2.3

$$
\mathbb{P}\left(T_{\omega}^{(x)}>t_{1}\right) \leq \mathbb{P}\left(\cap_{i=1}^{x}\left\{T_{i, \omega}>t_{1}\right\}\right)=\prod_{i=1}^{x} \mathbb{P}\left(T_{i, \omega}>t_{1}\right) \rightarrow\left(1-s_{\xi}\right)^{x},
$$

and

$$
\mathbb{P}\left(T_{\omega}^{(x)}>t_{1}\right) \geq \mathbb{P}\left(\cap_{i=1}^{x}\left\{T_{i, \frac{\omega}{x}}>t_{1}\right\}\right)=\prod_{i=1}^{x} \mathbb{P}\left(T_{i, \frac{\omega}{x}}>t_{1}\right) \rightarrow\left(1-s_{\xi}\right)^{x}
$$

\section{Exploring the graph}

We extend the Breadth First Search (BFS) graph exploration process of $\overrightarrow{\mathbb{G}}_{n}$ defined in Cai and Perarnau (2020).

For $\mathcal{I} \subseteq[n]$, let $\mathcal{E}^{ \pm}(\mathcal{I})$ be the set of heads/tails incident to the nodes in $\mathcal{I}$. Let $\mathcal{E}^{ \pm}:=\mathcal{E}^{ \pm}([n])$. For $\mathcal{X} \subseteq \mathcal{E}^{ \pm}$, let $\mathcal{V}(\mathcal{X})$ be the set of nodes incident to $\mathcal{X}$. Let $H$ be a partial pairing of half edges in $\mathcal{E}^{ \pm}$. Let $\mathcal{P}^{ \pm}(H) \subseteq \mathcal{E}^{ \pm}$be the set of heads/tails which are paired in $H$. Let $\mathcal{V}(H)=\mathcal{V}\left(\mathcal{P}^{ \pm}(H)\right)$. Let $\mathcal{F}^{ \pm}(H):=\mathcal{E}^{ \pm}(\mathcal{V}(H)) \backslash \mathcal{P}^{ \pm}(H)$ be the unpaired heads/tails which are incident to $\mathcal{V}(H)$. Let $E_{H}$ denote the event that $H$ is part of $\overrightarrow{\mathbb{G}}_{n}$. We will explore the graph conditioning on $E_{H}$.

We start from an arbitrary set $\mathcal{X}^{+}$of unpaired tails. In this process, we create random pairings of half-edges one by one and keep each half-edge in exactly one of the four states - active, paired, fatal or undiscovered. Let $\mathcal{A}_{i}^{ \pm}, \mathcal{P}_{i}^{ \pm}, \mathcal{F}_{i}^{ \pm}$and $\mathcal{U}_{i}^{ \pm}$denote the set of heads/tails in the four states respectively after the $i$-th pairing of half-edges. Initially, let

$$
\mathcal{A}_{0}^{+}=\mathcal{X}^{+}, \mathcal{A}_{0}^{-}=\mathcal{E}^{-}\left(\mathcal{V}\left(\mathcal{X}^{+}\right)\right), \mathcal{P}_{0}^{ \pm}=\mathcal{P}^{ \pm}(H), \mathcal{F}_{0}^{ \pm}=\mathcal{F}^{ \pm}(H), \mathcal{U}_{0}^{ \pm}=\mathcal{E}^{ \pm} \backslash\left(\mathcal{A}_{0}^{ \pm} \cup \mathcal{P}_{0}^{ \pm} \cup \mathcal{F}_{0}^{ \pm}\right)
$$

Then set $i=1$ and proceed as follows:

(i) Let $e_{i}^{+}$be one of the tails which became active earliest in $\mathcal{A}_{i-1}^{+}$. 
(ii) Pair $e_{i}^{+}$with a head $e_{i}^{-}$chosen uniformly at random from $\mathcal{E}^{-} \backslash \mathcal{P}_{i-1}^{-}$. Let $\mathcal{P}_{i}^{ \pm}=\mathcal{P}_{i-1}^{ \pm} \cup\left\{e_{i}^{ \pm}\right\}$.

(iii) If $e_{i}^{-} \in \mathcal{F}_{i-1}^{-}$, then terminate; if $e_{i}^{-} \in \mathcal{A}_{i-1}^{-}$, then $\mathcal{A}_{i}^{ \pm}=\mathcal{A}_{i-1}^{ \pm} \backslash\left\{e_{i}^{ \pm}\right\}$; and if $e_{i}^{-} \in \mathcal{U}_{i-1}^{-}$, then $\mathcal{A}_{i}^{ \pm}=\left(\mathcal{A}_{i-1}^{ \pm} \cup \mathcal{E}^{ \pm}\left(v_{i}\right)\right) \backslash\left\{e_{i}^{ \pm}\right\}$where $v_{i}=\mathcal{V}\left(e_{i}^{-}\right)$.

(iv) If $\mathcal{A}_{i}^{+}=\emptyset$ terminate; otherwise, $\mathcal{F}_{i}^{ \pm}=\mathcal{F}_{i-1}^{ \pm}, \mathcal{U}_{i}^{ \pm}=\mathcal{E}^{ \pm} \backslash\left(\mathcal{A}_{i}^{ \pm} \cup \mathcal{P}_{i}^{ \pm} \cup \mathcal{F}_{i}^{ \pm}\right), i=i+1$ and go to (i).

Let $F_{\mathcal{X}^{+}}(0)$ be a forest with $\left|\mathcal{X}^{+}\right|$isolated nodes corresponding to $\mathcal{X}^{+}$. Given $F_{\mathcal{X}^{+}}(i-1), F_{\mathcal{X}^{+}}(i)$ is constructed as follows: if $e_{i}^{-} \in \mathcal{U}_{i-1}^{-}$, then construct $F_{\mathcal{X}^{+}}(i)$ from $F_{\mathcal{X}^{+}}(i-1)$ by adding $\left|\mathcal{E}^{+}\left(v_{i}\right)\right|$ child nodes to the node representing $e_{i}^{+}$, each of which representing a tail in $\mathcal{E}^{+}\left(v_{i}\right)$; otherwise, let $F_{\mathcal{X}^{+}}(i)=F_{\mathcal{X}^{+}}(i-1)$. While $F_{\mathcal{X}^{+}}(i)$ is an unlabelled forest, its nodes correspond to the tails in $\left(\mathcal{P}_{i}^{+} \backslash \mathcal{P}_{0}^{+}\right) \cup \mathcal{A}_{i}^{+}$. So we can assign a label paired or active to each node of $F_{\mathcal{X}^{+}}(i)$.

For half-edges $e_{1}$ and $e_{2}$, we define the distance from $e_{1}$ to $e_{2}$, denoted by $\operatorname{dist}\left(e_{1}, e_{2}\right)$, to be the length of the shortest path from $v\left(e_{1}\right)$ to $v\left(e_{2}\right)$ which starts with the edge containing $e_{1}$ if $e_{1}$ is a tail, and which ends with the edge containing $e_{2}$ if $e_{2}$ is head. For two sets of half-edges $\mathcal{E}_{1}$ and $\mathcal{E}_{2}$, we define their distance by

$$
\operatorname{dist}\left(\mathcal{E}_{1}, \mathcal{E}_{2}\right):=\min _{e_{1} \in \mathcal{E}_{1}, e_{2} \in \mathcal{E}_{2}} \operatorname{dist}\left(e_{1}, e_{2}\right) .
$$

If $i_{t}$ is the last step where a tail at distance $t$ from $\mathcal{X}^{+}$is paired, then $F_{\mathcal{X}}\left(i_{t}\right)$ satisfies: (i) the height is $t$; (ii) the set of active nodes is the $t$-th level. We call a rooted forest $F$ incomplete if it satisfies (i)-(ii). We let $p(F)$ be the number of paired nodes in $F$.

3.1. Size biased distributions. We recall some notation from Cai and Perarnau (2020). The in- and out-size biased distributions of $D_{n}$ and $D$ are defined

$$
\begin{gathered}
\mathbb{P}\left(\left(D_{n}\right)_{\text {in }}=(k-1, \ell)\right)=\frac{k n_{k, \ell}}{m_{n}}, \quad \mathbb{P}\left(\left(D_{n}\right)_{\text {out }}=(k, \ell-1)\right)=\frac{\ell n_{k, \ell}}{m_{n}}, \\
\mathbb{P}\left(D_{\text {in }}=(k-1, \ell)\right)=\frac{k \lambda_{k, \ell}}{\lambda}, \quad \mathbb{P}\left(D_{\text {out }}=(k, \ell-1)\right)=\frac{\ell \lambda_{k, \ell}}{\lambda} .
\end{gathered}
$$

Then, by (i) of Condition 1.1, $\left(D_{n}\right)_{\text {in }} \rightarrow D_{\text {in }}$ and $\left(D_{n}\right)_{\text {out }} \rightarrow D_{\text {out }}$, and by (iii) of Condition 1.1,

$$
\lim _{n \rightarrow \infty} \mathbb{E}\left[\left(D_{n}\right)_{\text {in }}^{+}\right]=\lim _{n \rightarrow \infty} \mathbb{E}\left[\left(D_{n}\right)_{\text {out }}^{-}\right]=\mathbb{E}\left[D_{\text {in }}^{+}\right]=\mathbb{E}\left[D_{\text {out }}^{-}\right]=\frac{\mathbb{E}\left[D^{+} D^{-}\right]}{\lambda}=\nu .
$$

Let $s_{n+}, s_{n-}, s_{+}$and $s_{-}$be the survival probabilities of the branching processes with distribution $\left(D_{n}\right)_{\text {in }}^{+},\left(D_{n}\right)_{\text {out }}^{-}, D_{\text {in }}^{+}$and $D_{\text {out }}^{-}$respectively. Then as we have shown in Cai and Perarnau (2020), $s_{n \pm} \rightarrow s_{ \pm}$.

3.2. Coupling with branching processes. Consider the probability distribution $Q_{n}:=\left(D_{n}\right)_{\text {in }}^{+}$which satisfies for all $\ell \geq 0$,

$$
\mathbb{P}\left(Q_{n}=\ell\right)=q_{n, \ell}:=\frac{\sum_{k \geq 1} k n_{k, \ell}}{m_{n}} .
$$

In Cai and Perarnau (2020, Section 3), it has been shown that $Q_{n} \rightarrow D_{\text {in }}^{+}$in distribution and in expectation. In particular, by (3.5) $\mathbb{E}\left[Q_{n}\right] \rightarrow \mathbb{E}\left[D_{\text {in }}^{+}\right]=\nu$. Also in Cai and Perarnau (2020), we showed that the exploration process starting from one tail can be approximated by a branching process with offspring distribution $Q_{n}$. Similarly, the extended exploration process starting from $\mathcal{X}^{+}$can be approximated by $\left|\mathcal{X}^{+}\right|$independent branching processes with offspring distribution $Q_{n}$.

For $\beta \in(0,1 / 10)$, consider the distributions $Q_{n}^{\downarrow}=Q_{n}^{\downarrow}(\beta)$ and $Q_{n}^{\uparrow}=Q_{n}^{\uparrow}(\beta)$ defined by

$$
\begin{aligned}
& \mathbb{P}\left(Q_{n}^{\downarrow}=\ell\right)=q_{n, \ell}^{\downarrow}:= \begin{cases}c^{\downarrow} q_{n, \ell} & \text { if } q_{n, \ell} \geq n^{-2 \beta} \text { and } \ell \leq n^{\beta} \\
0 & \text { otherwise }\end{cases} \\
& \mathbb{P}\left(Q_{n}^{\uparrow}=\ell\right)=q_{n, \ell}^{\uparrow}:= \begin{cases}c^{\uparrow} q_{n, \ell} & \ell \geq 1 \\
c^{\uparrow} q_{n, 0}+n^{-1 / 2+2 \beta} & \ell=0\end{cases}
\end{aligned}
$$


where $c^{\downarrow}$ and $c^{\uparrow}$ are normalising constants.

Let $\mathrm{GW}_{\xi}^{(x)}=\left(\mathrm{GW}_{1, \xi}, \ldots, \mathrm{GW}_{x, \xi}\right)$ be $x$ independent Galton-Watson trees with offspring distribution $\xi$. Let $F=\left(T_{1}, \ldots, T_{x}\right)$ be an incomplete forest. Let $\mathrm{GW}_{\xi}^{(x)} \cong F$ denote that for every $i \in[x]$, $T_{i}$ is a root subtree of $\mathrm{GW}_{i, \xi}$ and all paired nodes of $T_{i}$ have the same degree in $\mathrm{GW}_{i, \xi}$.

The following lemma is a straightforward extension of Cai and Perarnau (2020, Lemma 5.3) and we omit its proof:

Lemma 3.1. Let $\beta \in(0,1 / 10)$ and let $H$ be a partial pairing with $|\mathcal{V}(H)| \leq n^{1-6 \beta}$. Let $\mathcal{X}^{+} \subset \mathcal{E}^{+}$ with $\left|\mathcal{X}^{+}\right|=x$. For every incomplete forest $F$ with $p(F) \leq n^{\beta}$, we have

$$
(1+o(1)) \mathbb{P}\left(\mathrm{GW}_{Q_{n}^{\downarrow}(\beta)}^{(x)} \cong F\right) \leq \mathbb{P}\left(F_{\mathcal{X}^{+}}(p(F))=F \mid E_{H}\right) \leq(1+o(1)) \mathbb{P}\left(\mathrm{GW}_{Q_{n}^{\uparrow}(\beta)}^{(x)} \cong F\right) .
$$

\section{Expansion probability}

Let $\mathcal{N}_{t}^{ \pm}\left(\mathcal{X}^{ \pm}\right)$and $\mathcal{N}_{\leq t}^{ \pm}\left(\mathcal{X}^{ \pm}\right)$be the sets of heads/tails at distance $t$ and at most $t$ from $\mathcal{X}^{ \pm} \subseteq \mathcal{E}^{ \pm}$ respectively. From now on, let

$$
\omega:=\log ^{6} n, \quad t_{0}:=\log _{\nu} \omega .
$$

Let $t_{\omega}\left(\mathcal{X}^{ \pm}\right)$be the expansion time of $\mathcal{X}^{ \pm}$defined as

$$
t_{\omega}\left(\mathcal{X}^{ \pm}\right):=\inf \left\{t \geq 1:\left|\mathcal{N}_{t}^{ \pm}\left(\mathcal{X}^{ \pm}\right)\right| \geq \omega\right\} \text {. }
$$

For brevity, we write $\mathcal{N} \leq \omega\left(\mathcal{X}^{ \pm}\right)=\cup_{t=1}^{t_{\omega}} \mathcal{N}_{t}^{ \pm}\left(\mathcal{X}^{ \pm}\right)$.

Given $H$ a partial pairing of $\mathcal{E}^{ \pm}$and $\mathcal{X}^{ \pm} \subseteq \mathcal{E}^{ \pm}$and $\varepsilon>0$, we consider the following two events:

$$
\begin{aligned}
& A_{1}\left(\mathcal{X}^{ \pm}, \varepsilon\right):=\left\{t_{\omega}\left(\mathcal{X}^{ \pm}\right) \leq(1+\varepsilon) t_{0}\right\} . \\
& A_{2}\left(\mathcal{X}^{ \pm}, H\right):=\left\{\mathcal{N}^{\leq \omega}\left(\mathcal{X}^{ \pm}\right) \cap \mathcal{F}^{ \pm}(H)=\emptyset\right\} .
\end{aligned}
$$

(Note that the second event above is only defined when we are conditioning on $E_{H}$.) The first lemma in this section shows that the probability that both these events happen is close to the survival probability of a branching process.

Lemma 4.1. Assume that $\nu>1$. Fix $x \in \mathbb{N}, \varepsilon \in(0,1 / 2)$ and $\gamma \in(0,1)$. Then uniformly for all choices of partial pairing $H$ and $\mathcal{X}^{ \pm} \subseteq \mathcal{E}^{ \pm}$with $|\mathcal{V}(H)| \leq n^{1-\gamma},\left|\mathcal{X}^{ \pm}\right|=x$, as $n \rightarrow \infty$,

$$
\mathbb{P}\left(A_{1}\left(\mathcal{X}^{ \pm}, \varepsilon\right) \cap A_{2}\left(\mathcal{X}^{ \pm}, H\right) \mid E_{H}\right)=(1+o(1))\left(1-\rho_{ \pm}^{x}\right) .
$$

Proof: Let $\mathcal{F}_{x, t, \omega}$ be the class of incomplete forests $F$ with $x$ trees, height $t$ and such that only the last level has at least $\omega$ nodes. Let $t_{1}=\left\lfloor(1+\varepsilon) t_{0}\right\rfloor$. For $t \leq t_{1}$ and $F \in \mathcal{F}_{x, t, \omega}$, we have $(t-1) \leq p(F) \leq x \omega t=O\left(\log ^{7} n\right)$. Let $\beta=\gamma / 100$. Let $X_{1, t}^{\uparrow}, \ldots, X_{x, t}^{\uparrow}$ be the sizes of the $t$ th generation of $x$ iid branching processes with offspring distribution $Q_{n}^{\uparrow}(\beta)$ and let $s_{+n}^{\uparrow}$ be the survival probability of each one. Since $Q_{n}^{\uparrow} \rightarrow D_{\text {in }}^{+}$in distribution, we have $s_{+n}^{\uparrow} \rightarrow s_{+}=1-\rho_{+}>0$.

Let $T_{\omega}^{\uparrow}=\inf \left\{t \geq 1: \sum_{i=1}^{x} X_{i, t}^{\uparrow} \geq \omega\right\}$. By Corollary 2.4 and Lemma 3.1, the LHS of (4.4) is

$$
\begin{aligned}
\sum_{t=1}^{t_{1}} \sum_{j=t-1}^{\lfloor x \omega t\rfloor} \sum_{\substack{F \in \mathcal{F}_{x, t, \omega} \\
p(F)=j}} \mathbb{P}\left(F_{\mathcal{X}^{+}}(x)=F \mid E_{H}\right) & \leq(1+o(1)) \sum_{t=1}^{t_{1}} \sum_{j=t-1}^{\lfloor x \omega t\rfloor} \sum_{\substack{F \in \mathcal{F}_{x, t, \omega} \\
p(F)=j}} \mathbb{P}\left(\mathrm{GW}_{Q_{n}^{\uparrow}(\beta)} \cong F\right) \\
& =(1+o(1)) \mathbb{P}\left(T_{\omega}^{\uparrow} \leq t_{1}\right) \\
& =(1+o(1))\left(1-\left(1-s_{+n}^{\uparrow}\right)^{x}\right) \\
& =(1+o(1))\left(1-\rho_{+}^{x}\right),
\end{aligned}
$$

where we used that $\nu>0$ implies $\rho_{ \pm}<1$. The lower bound follows from a similar argument with $Q_{n}^{\uparrow}$ replaced by $Q_{n}^{\downarrow}$. 
Our next lemma shows that when two sets of tails $\mathcal{X}_{1}^{+}$and $\mathcal{X}_{2}^{+}$satisfy that $\left|\mathcal{X}_{1}^{+}\right|\left|\mathcal{X}_{2}^{-}\right|$is small, then they are unlikely to be too close. We omit the proof since it follows from an easy adaptation of the proof in Cai and Perarnau (2020, Proposition 7.2).

Lemma 4.2. Assume that $\nu>1$. Fix $\varepsilon \in(0,1 / 2)$ and $\gamma \in(0,1)$. Then uniformly for all choices of partial pairing $H$ and $\mathcal{X}_{1}^{+}, \mathcal{X}_{2}^{+} \subseteq \mathcal{E}^{+}$with $|\mathcal{V}(H)| \leq n^{1-\gamma}$ and $\left|\mathcal{X}_{1}^{+}\right|\left|\mathcal{X}_{2}^{+}\right| \leq \omega \sqrt{n}$, we have

$$
\mathbb{P}\left(\operatorname{dist}\left(\mathcal{X}_{1}^{+}, \mathcal{X}_{2}^{+}\right) \leq\left(\frac{1}{2}-\varepsilon\right) \log _{\nu} n \mid E_{H}\right)=o\left(n^{-\varepsilon / 2}\right) .
$$

The previous lemma allows us to remove $A_{2}\left(\mathcal{X}^{ \pm}, H\right)$ in Lemma 4.1.

Lemma 4.3. Assume that $\nu>1$. Fix $x^{ \pm} \in \mathbb{N}$ and $\varepsilon \in(0,1 / 2)$. Then uniformly for all choices of partial pairing $H$ and $\mathcal{X}^{ \pm} \subseteq \mathcal{E}^{ \pm}$with $|\mathcal{V}(H)|=o\left(\omega^{2}\right),\left|\mathcal{X}^{ \pm}\right|=x^{ \pm}$, we have, as $n \rightarrow \infty$,

$$
\begin{aligned}
\mathbb{P}\left(A_{1}\left(\mathcal{X}^{ \pm}, \varepsilon\right) \mid E_{H}\right) & =(1+o(1))\left(1-\rho_{ \pm}^{x^{ \pm}}\right), \\
\mathbb{P}\left(A_{1}\left(\mathcal{X}^{+}, \varepsilon\right) \cap A_{1}\left(\mathcal{X}^{-}, \varepsilon\right) \mid E_{H}\right) & =(1+o(1))\left(1-\rho_{-}^{x^{-}}\right)\left(1-\rho_{+}^{x^{+}}\right) .
\end{aligned}
$$

Proof: We will prove (4.7) for $\mathcal{X}^{+}$; a similar argument works for $\mathcal{X}^{-}$. Let

$$
E_{1}=A_{1}\left(\mathcal{X}^{+}, \varepsilon\right), \quad E_{2}=A_{2}\left(\mathcal{X}^{+}, H\right), \quad E_{3}=A_{1}\left(\mathcal{X}^{-}, \varepsilon\right) .
$$

Note that the event $E_{2}$ happens if and only if $\operatorname{dist}\left(\mathcal{X}^{+}, \mathcal{F}^{+}(H)\right)>t_{\omega}\left(\mathcal{X}^{+}\right)$.

By Lemma 4.1, the LHS of (4.7) equals

$$
\begin{aligned}
\mathbb{P}\left(E_{1} \mid E_{H}\right) & =\mathbb{P}\left(E_{1} \cap E_{2} \mid E_{H}\right)+\mathbb{P}\left(E_{1} \cap E_{2}^{c} \mid E_{H}\right) \\
& =(1+o(1))\left(1-\rho_{+}^{x^{+}}\right)+\mathbb{P}\left(E_{1} \cap E_{2}^{c} \mid E_{H}\right) .
\end{aligned}
$$

Since $|\mathcal{V}(H)|=o\left(\omega^{2}\right)$, by Cai and Perarnau (2020, Lemma 2.2) we have $\left|\mathcal{E}^{+}(H)\right|=o(\omega \sqrt{n})$. By Lemma 4.2, for $\delta<1 / 2$,

$$
\begin{aligned}
\mathbb{P}\left(E_{1} \cap E_{2}^{c} \mid E_{H}\right) & \leq \mathbb{P}\left(\operatorname{dist}\left(\mathcal{X}^{+}, \mathcal{F}^{+}(H)\right) \leq 4 t_{0} \mid E_{H}\right) \\
& \leq \mathbb{P}\left(\operatorname{dist}\left(\mathcal{X}^{+}, \mathcal{F}^{+}(H)\right) \leq\left(\frac{1}{2}-\delta\right) \log n \mid E_{H}\right)=o(1) .
\end{aligned}
$$

This proves (4.7) for $\mathcal{X}^{+}$and we are left with (4.8).

Let $\mathcal{H}$ be the set of all possible partial pairings in $\mathcal{N} \leq \omega\left(\mathcal{X}^{+}\right)$such that $E_{1} \cap E_{H}$ happens. Then $H^{\prime} \in \mathcal{H}$ implies that $\left|\mathcal{V}\left(H^{\prime}\right)\right|,\left|\mathcal{V}\left(H^{\prime} \cup H\right)\right|=o\left(\omega^{2}\right)$, uniformly over all choices of $H^{\prime}$. Using Lemma 4.1 again, we have

$$
\begin{aligned}
\mathbb{P}\left(E_{1} \cap E_{3} \mid E_{H}\right) & =\sum_{H^{\prime} \in \mathcal{H}} \mathbb{P}\left(E_{3} \mid E_{H \cup H^{\prime}}\right) \mathbb{P}\left(E_{H \cup H^{\prime}} \mid E_{H}\right) \\
& =\sum_{H^{\prime} \in \mathcal{H}}(1+o(1))\left(1-\rho_{-}^{x^{-}}\right) \mathbb{P}\left(E_{H^{\prime} \cup H} \mid E_{H}\right) \\
& =(1+o(1))\left(1-\rho_{-}^{x^{-}}\right) \mathbb{P}\left(E_{1} \mid E_{H}\right) \\
& =(1+o(1))\left(1-\rho_{-}^{x^{-}}\right)\left(1-\rho_{+}^{x^{+}}\right) .
\end{aligned}
$$

Unsurprisingly, Lemma 4.3 can be extended to a fixed number of pairs of head-sets and tail-sets:

Lemma 4.4. Assume that $\nu>1$. Fix $i, x_{1}^{ \pm}, \ldots, x_{i}^{ \pm} \in \mathbb{N}$ and $\varepsilon \in(0,1 / 2)$. Then uniformly for all disjoint sets of tails $\left(\mathcal{X}_{1}^{+}, \ldots, \mathcal{X}_{i}^{+}\right)$and disjoint sets of heads $\left(\mathcal{X}_{1}^{-}, \ldots, \mathcal{X}_{i}^{-}\right)$with $\left|\mathcal{X}_{j}^{ \pm}\right|=x_{j}^{ \pm}$for $j \in[i]$, we have, as $n \rightarrow \infty$,

$$
\mathbb{P}\left(\cap_{j=1}^{i}\left(A_{1}\left(\mathcal{X}^{+}, \varepsilon\right) \cap A_{1}\left(\mathcal{X}^{-}, \varepsilon\right)\right)\right)=(1+o(1)) \prod_{j=1}^{i}\left(1-\rho_{-}^{x_{j}^{-}}\right)\left(1-\rho_{+}^{x_{j}^{+}}\right) .
$$


Proof: We prove it by induction. The case $i=1$ follows by Lemma 4.3 with $H$ an empty pairing.

Let $E_{j}$ denote the event in the LHS of (4.13). Assume that the lemma holds for some $i \geq 1$. Let $\mathcal{H}$ be the sets of all possible partial pairings in $\cup_{j=1}^{i}\left(\mathcal{N} \leq \omega\left(\mathcal{X}_{j}^{+}\right) \cup \mathcal{N} \leq \omega\left(\mathcal{X}_{j}^{-}\right)\right)$compatible with $E_{i}$. If $H \in \mathcal{H}$, then $|\mathcal{V}(H)|=o\left(\omega^{2}\right)$. Using Lemma 4.1 as in (4.13), we conclude

$$
\mathbb{P}\left(E_{i+1}\right)=\sum_{H \in \mathcal{H}} \mathbb{P}\left(E_{i+1} \mid E_{H}\right) \mathbb{P}\left(E_{H}\right)=(1+o(1))\left(1-\rho_{-}^{x_{i+1}^{-}}\right)\left(1-\rho_{+}^{x_{i+1}^{+}}\right) \mathbb{P}\left(E_{i}\right) .
$$

The last lemma shows that expansions are unlikely to happen very late.

Lemma 4.5. Assume that $\nu>1$. Fix $x^{ \pm} \in \mathbb{N}$ and $\varepsilon \in(0,1 / 2)$. Then uniformly for all choices of $\mathcal{X}^{ \pm} \subseteq \mathcal{E}^{ \pm}$with $\left|\mathcal{X}^{ \pm}\right|=x^{ \pm}$, as $n \rightarrow \infty$,

$$
\mathbb{P}\left(t_{\omega}\left(\mathcal{X}^{ \pm}\right) \in\left((1+\varepsilon) t_{0}, \infty\right)\right)=o(1)
$$

Proof: Let $t_{1}=\left\lfloor(1+\varepsilon) t_{0}\right\rfloor$. Note that

$$
\mathbb{P}\left(t_{\omega}\left(\mathcal{X}^{ \pm}\right) \in\left(t_{1}, \infty\right)\right) \leq \sum_{e^{ \pm} \in \mathcal{X}^{ \pm}} \mathbb{P}\left(t_{\omega}\left(e^{ \pm}\right) \in\left(t_{1}, \infty\right)\right)
$$

Thus we may assume that $\mathcal{X}^{ \pm}=\left\{e^{ \pm}\right\}$. Let $X_{t}^{\uparrow}$ be the size of the $t$-th generation of a branching process with offspring distribution $Q_{n}^{\uparrow}(\beta)$ for some $\beta \in(0,1 / 10)$. Let $T_{\omega}=\inf \left\{t \geq 1: X_{t}^{\uparrow} \geq \omega\right\}$. Then it follows from Cai and Perarnau (2020, Theorem 3.4) that there exist constants $C>0$ and $\hat{\nu} \in(0,1)$ such that

$$
\mathbb{P}\left(T_{\omega} \in\left(t_{1}, \infty\right)\right) \leq \mathbb{P}\left(\cap_{t=0}^{t_{1}}\left\{X_{t} \in(0, \omega)\right\}\right) \leq C(1+o(1) \hat{\nu})^{(1+\varepsilon) t_{0}-(1+o(1)) t_{0}}=o(1) .
$$

By the same argument as in Lemma 4.1, this implies $\mathbb{P}\left(t_{\omega}\left(e^{ \pm}\right) \in\left(t_{1}, \infty\right)\right)=o(1)$.

\section{Expectation and variance}

Lemma 5.1. Assume that $\nu>1$. Let

$$
\mathcal{L}:=\left\{v \in[n]: t_{\omega}\left(\mathcal{E}^{+}(v)\right)<\infty, t_{\omega}\left(\mathcal{E}^{-}(v)\right)<\infty\right\} .
$$

Then

$$
\frac{\mathbb{E}[|\mathcal{L}|]}{n} \rightarrow \eta, \quad \frac{\mathbb{E}\left[|\mathcal{L}|^{2}\right]}{n^{2}} \rightarrow \eta^{2}
$$

where $\eta$ is defined as in (1.7). Thus, $|\mathcal{L}| / n \rightarrow \eta$ in probability.

Proof: As $\rho_{ \pm}<1$ and $\sum_{i, j \geq 0} \lambda_{i, j}=1$, we have $\eta \in(0,1)$. Fix $\varepsilon \in(0,1 / 2)$. Define

$$
\mathcal{L}(\varepsilon):=\left\{v \in[n]: t_{\omega}\left(\mathcal{E}^{+}(v)\right)<(1+\varepsilon) t_{0}, t_{\omega}\left(\mathcal{E}^{-}(v)\right)<(1+\varepsilon) t_{0}\right\} .
$$

and note that $\mathcal{L}(\varepsilon) \subseteq \mathcal{L}$. Given $v \in[n]$ with $i$ heads and $j$ tails, it follows from Lemma 4.4 that

$$
p_{i, j}:=\mathbb{P}(v \in \mathcal{L}(\varepsilon))=(1+o(1))\left(1-\rho_{-}^{i}\right)\left(1-\rho_{+}^{j}\right) .
$$

Since there are $n_{i, j}$ such nodes, by (i) of Condition 1.1,

$$
\frac{\mathbb{E}[|\mathcal{L}(\varepsilon)|]}{n}=\sum_{i, j \geq 0} \frac{n_{i, j}}{n} p_{i, j}=\sum_{i, j \geq 0}(1+o(1)) \lambda_{i, j}\left(1-\rho_{-}^{i}\right)\left(1-\rho_{+}^{j}\right) \rightarrow \eta .
$$

To see that the sum above converges to $\eta$, note that $\sum_{i, j \geq 0} \frac{n_{i, j}}{n}=1$ and $p_{i, j} \leq 1$. Thus we can apply the dominated convergence theorem by considering the double sum as an integral over $\mathbb{Z}_{\geq 0}^{2}$ with respect to the counting measure. Lemma 4.5 implies $\mathbb{P}(v \in \mathcal{L} \backslash \mathcal{L}(\varepsilon))=o(1)$. Thus $\mathbb{E}[|\mathcal{L} \backslash \mathcal{L}(\varepsilon)|]=o(n)$, which finishes the proof for the expectation. 
Given distinct $v_{1}, v_{2} \in[n]$ with degrees $\left(i_{1}, j_{1}\right)$ and $\left(i_{2}, j_{2}\right)$, again by Lemma 4.4

$$
p_{i_{1}, j_{1}, i_{2}, j_{2}}:=\mathbb{P}\left(\left\{v_{1} \in \mathcal{L}(\varepsilon)\right\} \cap\left\{v_{2} \in \mathcal{L}(\varepsilon)\right\}\right)=(1+o(1)) \prod_{r=1}^{2}\left(1-\rho_{-}^{i_{r}}\right)\left(1-\rho_{+}^{j_{r}}\right) .
$$

By the same convergence argument used in (5.5), we have

$$
\frac{\mathbb{E}\left[|\mathcal{L}(\varepsilon)|^{2}\right]}{n^{2}}=o(1)+\sum_{i_{1}, j_{1}, i_{2}, j_{2} \geq 0} \frac{n_{i_{1}, j_{1}} n_{i_{2}, j_{2}}}{n^{2}} p_{i_{1}, j_{1}, i_{2}, j_{2}} \rightarrow \eta^{2}
$$

As $\mathbb{E}[|\mathcal{L} \backslash \mathcal{L}(\varepsilon)|]=o(n)$, the following concludes the proof for the second moment:

$$
\mathbb{E}\left[|\mathcal{L}|^{2}-|\mathcal{L}(\varepsilon)|^{2}\right]=\mathbb{E}[(|\mathcal{L}|-|\mathcal{L}(\varepsilon)|)(|\mathcal{L}|+|\mathcal{L}(\varepsilon)|)] \leq 2 n \mathbb{E}[|\mathcal{L} \backslash \mathcal{L}(\varepsilon)|]=o\left(n^{2}\right)
$$

Lemma 5.2. Assume that $\nu>1$. Let $\mathcal{L}_{e}$ be the set of edges whose both endpoints are in $\mathcal{L}$. Then

$$
\frac{\mathbb{E}\left[\left|\mathcal{L}_{e}\right|\right]}{n} \rightarrow \lambda s_{-} s_{+}, \quad \frac{\mathbb{E}\left[\left|\mathcal{L}_{e}\right|^{2}\right]}{n^{2}} \rightarrow\left(\lambda s_{-} s_{+}\right)^{2} .
$$

Thus $\left|\mathcal{L}_{e}\right| / n \rightarrow \zeta$ in probability.

Proof: We only sketch the proof since the argument is very similar to that of Lemma 5.1.

Given $v_{1}, v_{2} \in[n]$ with degrees $\left(i_{1}, j_{1}\right)$ and $\left(i_{2}, j_{2}\right)$ respectively, the number of edges $X_{v_{1}, v_{2}}$ from $v_{1}$ to $v_{2}$ satisfies $\mathbb{E}\left[X_{v_{1}, v_{2}}\right]=j_{1} i_{2} / m_{n}$. It follows from Lemma 4.4 that conditioning on $X_{v_{1}, v_{2}}$, the probability that both $v_{1}$ and $v_{2}$ are in $\mathcal{L}$ converges to $p_{v_{1} v_{2}}:=\left(1-\rho_{-}^{i_{1}}\right)\left(1-\rho_{+}^{j_{2}}\right)$. We have

$$
\begin{aligned}
\frac{\mathbb{E}\left[\left|\mathcal{L}_{e}\right|\right]}{n}= & \sum_{v_{1}, v_{2} \in[n]} \frac{\mathbb{E}\left[X_{v_{1}, v_{2}}\right] \cdot(1+o(1)) p_{v_{1}, v_{2}}}{n} \\
= & \sum_{i_{1}, j_{1}, i_{2}, j_{2} \geq 0}(1+o(1)) \frac{n_{i_{1}, j_{1}} n_{i_{2}, j_{2}}}{n} \frac{j_{1} i_{2}}{m_{n}}\left(1-\rho_{-}^{i_{1}}\right)\left(1-\rho_{+}^{j_{2}}\right) \\
& -\sum_{i_{1}, j_{1} \geq 0}(1+o(1)) \frac{n_{i_{1}, j_{1}}}{n} \frac{i_{1} j_{1}}{m_{n}}\left(1-\rho_{-}^{i_{1}}\right)\left(1-\rho_{+}^{j_{1}}\right) \\
= & \left(\frac{1}{\lambda} \sum_{i_{1}, j_{1} \geq 0} \sum_{i_{2}, j_{2} \geq 0}(1+o(1)) \lambda_{i_{1}, j_{1}} j_{1}\left(1-\rho_{-}^{i_{1}}\right) \cdot \lambda_{i_{2}, j_{2}} i_{2}\left(1-\rho_{+}^{j_{2}}\right)\right)-o(1) \\
\rightarrow & \lambda\left(1-\frac{1}{\lambda} \frac{\partial f}{\partial w}\left(\rho_{-}, 1\right)\right)\left(1-\frac{1}{\lambda} \frac{\partial f}{\partial z}\left(1, \rho_{+}\right)\right)=\lambda s_{-} s_{+} .
\end{aligned}
$$

The proof for the second moment is similar and we omit it.

\section{Proof of Theorem 1.2}

If $\nu>1$, it suffices to show that whp the set $\mathcal{L}$ defined in (5.1) exactly coincides with the largest sCC. Then (1.5) and (1.6) in Theorem 1.2 follow immediately from Lemma 5.1 and Lemma 5.2.

By Cai and Perarnau (2020, Proposition 7.2), uniformly for all $\mathcal{X}^{ \pm} \subseteq \mathcal{E}^{ \pm}$with $\left|\mathcal{X}^{ \pm}\right| \geq \omega$,

$$
\mathbb{P}\left(\operatorname{dist}\left(\mathcal{X}^{+}, \mathcal{X}^{-}\right)=\infty\right)=o\left(n^{-100}\right) \text {. }
$$

Since every node in $\mathcal{L}$ reaches at least $\omega$ other nodes and is reachable from at least $\omega$ other nodes, (6.1) implies that whp, for each pair of nodes $u, v \in \mathcal{L}$ there is path from $u$ to $v$. In other words, $\mathcal{L}$ is contained in a SCC whp. It remains to show that whp there is no other vertex in this SCC other than those in $\mathcal{L}$.

For an half-edge $e^{ \pm}$, define the event

$$
A_{3}\left(e^{ \pm}, t\right)=\cap_{r=1}^{t}\left\{0<\mathcal{N}_{r}\left(e^{ \pm}\right)<\omega\right\} .
$$


(In other words, $A_{3}\left(e^{ \pm}, t\right)$ is the event that the neighbourhood of $e^{ \pm}$remains small but non-empty until at least distance $t$.) By Cai and Perarnau (2020, Proposition 6.1), there exists a constant $\hat{\nu}_{ \pm} \in(0,1)$ such that for $t=\Theta(\log n)$,

$$
\mathbb{P}\left(A_{3}\left(e^{ \pm}, t\right)\right)=\hat{\nu}_{ \pm}^{(1+o(1)) t} .
$$

Thus, letting $t_{2}^{ \pm}=\left\lceil 2 \log _{1 / \hat{\nu}_{ \pm}}(n)\right\rceil$, we have

$$
\mathbb{P}\left(\cup_{e^{+} \in \mathcal{E}^{+}} \cup_{e^{-} \in \mathcal{E}^{-}}\left(A_{3}\left(e^{+}, t_{2}^{+}\right) \cup A_{3}\left(e^{-}, t_{2}^{-}\right)\right)\right) \leq\left(m_{n} n^{-3 / 2}\right)^{2}=o(1) .
$$

Therefore, whp, each node either belongs to $\mathcal{L}$, i.e., both its in- and out-neighbourhoods expand to size $\omega$, or it has no out-neighbourhood at distance $t_{2}^{+}$from it, or it has no in-neighbourhood at distance $t_{2}^{-}$to it. This implies that whp $\mathcal{L}$ is a SCC and that any other sCCs have size at most $\omega t_{2}^{ \pm}=O\left(\log ^{7} n\right)$. This concludes the proof of the supercritical case.

For the subcritical case, we first show the following lemma.

Lemma 6.1. Assume that $\nu<1$. Let $C_{n, \geq \ell}$ be the number of directed simple cycles in $\overrightarrow{\mathbb{G}}_{n}$ of length at least $\ell$. Then,

$$
\limsup _{n \rightarrow \infty} \mathbb{E}\left[C_{n, \geq 1}\right] \leq \log \left(\frac{1}{1-\nu}\right)
$$

Moreover, for any $\ell_{n} \rightarrow \infty$,

$$
\limsup _{n \rightarrow \infty} \mathbb{E}\left[C_{n, \geq \ell_{n}}\right]=0
$$

Proof: Let $C_{n, k}$ be the number of directed cycles of length $k \geq 1$. (If $k=1$, then $C_{n, 1}$ is the number of loops.) Let $v \in[n]$ with degrees $(i, j)$. By Cai and Perarnau (2020, Lemma 7.3) the expected number of simple paths of length $k$ from $\mathcal{E}^{+}(v)$ to $\mathcal{E}^{-}(v)$ is at most $(1+o(1)) i j \nu^{k-1} / m_{n}$. As each cycle of length $k$ is counted $k$ times, we have

$$
\mathbb{E}\left[C_{n, k}\right] \leq \frac{1}{k} \sum_{i, j \geq 0}(1+o(1)) \frac{n_{i, j} i j \nu^{k-1}}{m_{n}} \rightarrow \frac{\nu^{k}}{k} .
$$

We conclude that

$$
\begin{aligned}
& \limsup _{n \rightarrow \infty} \mathbb{E}\left[C_{n, \geq 1}\right]=\limsup _{n \rightarrow \infty} \sum_{k \geq 1} \mathbb{E}\left[C_{n, k}\right] \leq \sum_{k \geq 1} \frac{\nu^{k}}{k}=\log \left(\frac{1}{1-\nu}\right), \\
& \limsup _{n \rightarrow \infty} \mathbb{E}\left[C_{n, \geq \ell}\right]=\limsup _{n \rightarrow \infty} \sum_{k \geq \ell} \mathbb{E}\left[C_{n, k}\right] \leq \sum_{k \geq \ell} \frac{\nu^{k}}{k} \leq \frac{1}{\ell+1}\left(\frac{\nu}{1-\nu}\right)^{\ell},
\end{aligned}
$$

where the last inequality follows from the error bound on the Taylor approximation of $\log \left(\frac{1}{1-\nu}\right)$.

The above lemma shows that, for any $\ell_{n} \rightarrow \infty$, whp (i) there are at most $\ell_{n}$ cycles in $\overrightarrow{\mathbb{G}}_{n}$, and (ii) all cycles have length at most $\ell_{n}$. As any vertex in a SCC belongs to at least one cycle, it follows that any SCC has order at most $\ell_{n}^{2}$. This finishes the proof of the subcritical case.

Remark 6.2. In Cai and Devroye (2017), it was showed that the number of cycles outside the giant of a uniform random $k$-out digraph with $k \geq 2$ converges to a Poisson distribution. We believe that similar methods can be applied to derive that the law of $C_{n, \geq 1}$ converges to a Poisson distribution with mean $\log \left(\frac{1}{1-\nu}\right)$. 


\section{Binomial Random Digraphs}

The binomial random digraph $\mathbb{D}_{n, p}$ is a simple digraph on $[n]$ in which each ordered pair of nodes is connected with an arc independently at random with probability $p$, see Frieze and Karoński (2016, Chapter 12).

Although the degrees of nodes in $\mathbb{D}_{n, p}$ are random, conditioning on its degree sequence, $\mathbb{D}_{n, p}$ has the same probability to be any simple digraph with such a degree sequence. Thus we can study its properties through the directed configuration model. Using this method, we were able to show that the diameter of $\mathbb{D}_{n, p}$ converges in probability in Cai and Perarnau (2020, Theorem 9.5).

The same argument can be applied to determine the largest SCC in $\mathbb{D}_{n, p}$. Assuming that $n p \rightarrow \nu$, the degree of a uniform random node in $\mathbb{D}_{n, p}$ converges in distribution to two independent Poisson random variables with mean $\nu$. Thus, by Theorem 1.2 we recover the following result by Karp (1990):

Theorem 7.1. Assume that $n p \rightarrow \nu \neq 1$. Let $\rho$ be the smallest solution of $\rho=e^{-\nu(1-\rho)}$ on $(0,1]$. (In particular, if $\nu<1$ then $\rho=1$.) Let $\mathcal{G}_{n}$ be the largest strongly connected component in $\mathbb{D}_{n, p}$. Then

$$
\frac{v\left(\mathcal{G}_{n}\right)}{n} \rightarrow(1-\rho)^{2}, \quad \frac{e\left(\mathcal{G}_{n}\right)}{n} \rightarrow \nu(1-\rho)^{2},
$$

in probability.

The case $\nu=1$ has attracted some attention recently. Coulson (2019) determined the critical window of the model, and Goldschmidt and Stephenson (2019) showed convergence of the sequence of rescaled largest SCC within the critical window.

Pittel and Poole (2016) showed that in fact the joint distribution of $v\left(\mathcal{G}_{n}\right)$ and $e\left(\mathcal{G}_{n}\right)$ is asymptotically Gaussian in $\mathbb{D}_{n, p}$. It would be interesting to see if this holds in the directed configuration model.

\section{References}

Athreya, K. B. and Ney, P. E. Branching processes. Die Grundlehren der mathematischen Wissenschaften, Band 196. Springer-Verlag, New York-Heidelberg (1972). MR0373040.

Blanchet, J. and Stauffer, A. Characterizing optimal sampling of binary contingency tables via the configuration model. Random Structures Algorithms, 42 (2), 159-184 (2013). MR3019396.

Cai, X. S. and Devroye, L. The graph structure of a deterministic automaton chosen at random. Random Structures Algorithms, 51 (3), 428-458 (2017). MR3689339.

Cai, X. S. and Perarnau, G. The Diameter of the Directed Configuration Model. ArXiv Mathematics e-prints (2020). arXiv: 2003.04965.

Cooper, C. and Frieze, A. The size of the largest strongly connected component of a random digraph with a given degree sequence. Combin. Probab. Comput., 13 (3), 319-337 (2004). MR2056402.

Coulson, M. The Critical Window in Random Digraphs. ArXiv Mathematics e-prints (2019). arXiv: 1905.00624.

Donderwinkel, S. and Xie, Z. Universality for the Directed Configuration Model with Random Degrees: Metric Space Convergence of the Strongly Connected Components at Criticality. ArXiv Mathematics e-prints (2021). arXiv: 2105.11434.

Frieze, A. and Karoński, M. Introduction to random graphs. Cambridge University Press, Cambridge (2016). ISBN 978-1-107-11850-8. MR3675279.

Goldschmidt, C. and Stephenson, R. The Scaling Limit of a Critical Random Directed Graph. ArXiv Mathematics e-prints (2019). arXiv: 1905.05397.

Graf, A. On the Strongly Connected Components of Random Directed Graphs with given Degree Sequences. Ph.D. thesis, University of Waterloo (2016). http://hdl.handle.net/10012/10681. 
Janson, S. The probability that a random multigraph is simple. Combin. Probab. Comput., 18 (1-2), 205-225 (2009). MR2497380.

Karp, R. M. The transitive closure of a random digraph. Random Structures Algorithms, 1 (1), 73-93 (1990). MR1068492.

Pakes, A. G. Some limit theorems for the total progeny of a branching process. Advances in Appl. Probability, 3, 176-192 (1971). MR283892.

Pittel, B. and Poole, D. Asymptotic distribution of the numbers of vertices and arcs of the giant strong component in sparse random digraphs. Random Structures Algorithms, 49 (1), 3-64 (2016). MR3521273.

van der Hofstad, R. Random graphs and complex networks. Vol. 1. Cambridge Series in Statistical and Probabilistic Mathematics, [43]. Cambridge University Press, Cambridge (2017). ISBN 9781-107-17287-6. MR3617364. 